\title{
STUDY OF ASSOCIATION BETWEEN CHARACTERISTICS OF PATIENT AND LIKELIHOOD OF BENZODIAZEPINE USE IN PSYCHIATRY OUTPATIENT DEPARTMENT AT TERTIARY CARE HOSPITAL OF KATHMANDU.
}

\author{
Binod Raut ${ }^{1}$, Anjan Khadka², Pradeep Manandhar ${ }^{3}$, Kamal Kandel ${ }^{4}$
}

\begin{abstract}
${ }^{1}$ Department of Pharmacology, Kathmandu Medical College and Teaching Hospital (KMCTH), Duwakot, Bhaktapur, Nepal. ${ }^{2}$ Department of Pharmacology, Nepalese Army Institute of Health Sciences (NAIHS), College of Medicine, Sanobharyang, Kathmandu, Nepal. ${ }^{3}$ PDepartment of Psychiatry, Nepalese Army Institute of Health Sciences (NAIHS), College of Medicine, Sanobharyang, Kathmandu, Nepal.

${ }^{4}$ Department of Pharmacology, Kathmandu Medical College and Teaching Hospital (KMCTH), Duwakot, Bhaktapur, Nepal.
\end{abstract}

*Correspondence to: Dr Anjan Khadka, Assistant Professor, Department of Pharmacology, Nepalese Army Institute of Health Sciences (NAIHS), College of Medicine, Sanobharyang, Kathmandu, Nepal.E-mail: anjankhadka14@gmail.com

\begin{abstract}
Introduction: Benzodiazepines exert their pharmacological properties as hypnotics, anxiolytics, anticonvulsants and muscle relaxants. Benzodiazepines are clinically effective for a number of indication including the reduction of anxiety, the induction and maintenance of sleep, muscle relaxation. They have a range of well documented adverse effects that may outweigh the benefits in certain patient population including psychomotor impairment, development of tolerance and dependence, potential for abuse. Methods: It is a hospital based prospective cross-sectional study conducted in a psychiatry outpatient department of tertiary care hospital. All the patients attended to the outpatient department of psychiatry and prescribed with benzodiazepine were selected for study. Drug therapy details in medication chart review and clinical review in patients treated with benzodiazepines was analyzed to measure the utilization pattern of benzodiazepines in terms of patient characteristics (e.g. age, gender and marital status), occupation, education and diagnosis, number of prescription of benzodiazepines, their doses, frequency, routes of administration and duration of use in each patient. Results: Out of a total of 384 patients, 246 of the patients had been currently using at least one benzodiazepine. Prevalence of benzodiazepine use was $64 \%$. There was statistically significant association between benzodiazepines use with gender distribution, occupation and education. The prevalence of benzodiazepines use is relatively higher than that reported in the developed countries. Clonazepam was the most widely used benzodiazepine followed by lorazepam. Conclusion: The study evaluated the association between characteristics of patient and likelihood of benzodiazepines use. This will definitely help to optimize the drug therapy, improve the quality of care and reduce the negative outcomes in the usage of benzodiazepines.
\end{abstract}

Key words: Benzodiazepine, Patients, Psychiatry

\section{INTRODUCTION}

The importance of mental health has been gaining attention from various corner of society. The focus should also be given to the role of drugs in the treatment and prevention of mental illness. Among various drugs used in psychiatry, benzodiazepines are one of the commonest group which are prescribed all over the world. ${ }^{1}$

Benzodiazepine drugs were first introduced into clinical practice fifty years ago as anxiolytics and hypnotic agents. ${ }^{2}$ Benzodiazepines are clinically effective for a number of indications including the reduction of anxiety, the induction and maintenance of sleep, muscle relaxation, and the treatment and prevention of epileptic seizures. ${ }^{3,4}$ Though there is widespread concerns on benzodiazepine use for three decades regarding their unfavourable side effects profile as well as their propensity for dependence and potential for abuse, these drugs are still widely prescribed in most industrialized countries. ${ }^{5,6}$ Diazepam is reputedly one of the most 
widely prescribed drugs of all time. ${ }^{7}$ In addition, the benzodiazepines have questionable clinical effectiveness which leads to matter of concerns for health professionals and most importantly patients in prolonged use. ${ }^{8}$

Howeve, there are no such largescale studies on the rational use of benzodiazepines as individual based therapy. For that purpose, it is essential to know the association of drug usage and the population demographics. Though pharmacogenetic and pharmacogenomics data will be more appropriate for the individual tailored use of benzodiazepines, population demographics might be useful as the initiation towards the more scientific rationalized use of drug in the developing world like ours until the advancement of genomic research in our part of world. ${ }^{9}$

Population characteristics can yield data to complement prescribers since the prescription of drug is based on individual's requirement. ${ }^{10}$ Population studies assist the actual benzodiazepine users, the time and rationale of the initial prescription, and the number of months or years of use. Long-term use of benzodiazepines leads to physical and mental problems, so it is recommended for short-term use only. ${ }^{11}$ These factors lead to noncompliance in patients. It is therefore important to perform a study investigating the utilization patterns of benzodiazepines in different diseases in a given clinical setting. The estimation of beneficial and adverse effects of benzodiazepines should take into consideration their immediate and longterm consequences upon social, emotional and professional life. The establishment of significant association between several demographic pattern of patient and benzodiazepine use leads to the effective implentation of rational prescribing of benzodiazepines. The rational use of benzodiazepines has a long way to go which certainly includes training of prescribers, and informing and educating the patient as well as general population.

The present study aimed to assess the association between characteristics of patient and likelihood of benzodiazepines use at psychiatry outpatient department at tertiary care hospital of Kathmandu. In the wake of implementation of stricter and rational recommendation on the use of benzodiazepines, this study will help to define the boundary for the use of such drugs in individual patient.

\section{Materials and Methods}

The prospective cross-sectional study involving 384 patients based on non-probability sampling was conducted in the outpatient Department of psychiatry of tertiary care hospital from May 2016 to December 2016 after obtaining ethical approval from institutional ethical committee. Data has been recorded in customized proforma from the outpatient daily record sheets and reports. Outpatient department record sheets were analyzed for patient characteristics (e.g. age, gender and marital status), occupation, education and diagnosis, number of prescription of benzodiazepines, their doses, frequency, routes of administration and duration of use in each patient.

SPSS package version 20 was used for analysis. Results are expressed as mean standard deviation. The Chi-square test was carried out to determine the statistical significance of the differences between the prescription rates.

\section{Observations and Results}

During the study period, prescription of 384 patients attended at outpatient department of psychiatry were assessed. Among 384 patients, only 246 (64\%) of them were prescribed benzodiazepines.

\section{A. Age wise distribution}

The mean age of the patient attending psychiatry OPD was 37.05 years, ranging from minimum of 13 years to a maximum of 82 years. The highest numbers of patients attended to the psychiatry outpatient department were between the age group 30-39 years old (105 patients i.e. 27.34\%) which was followed by the age group $20-29$ years old (102 patients i.e. 26.56\%).

Although the highest number of patient attended were from the age group 30-39 years old, the highest prescribing rate of benzodiazepines were found among the age group between $50-59$ years old. There was no statistically significant association were found between benzodiazepines use and age distribution $(p$-value $=0.958)$ [Table 1]. Benzodiazepines were not prescribed to the age group $80-89$ years. 
Table 1: Association between age and the likelihood of benzodiazepines prescription

\begin{tabular}{|l|l|l|l|}
\hline \multicolumn{1}{|c|}{$\begin{array}{c}\text { Age group } \\
\text { (years) }\end{array}$} & \multicolumn{1}{|c|}{$\begin{array}{c}\text { Number (\%) of } \\
\text { patients }\end{array}$} & $\begin{array}{c}\text { Patients prescribed with } \\
\text { benzodiazepines (\%) }\end{array}$ & 'p' value \\
\hline $10-19$ & $28(7.29)$ & $12(42.85)$ \\
\hline $20-29$ & $102(26.56)$ & $54(52.94)$ \\
\hline $30-39$ & $105(27.34)$ & $71(67.61)$ & \\
\cline { 1 - 3 } 0 & $77(20.05)$ & $56(72.72)$ & \\
\cline { 1 - 3 } $0-49$ & $37(9.63)$ & $28(75.67)$ & \\
\hline $60-69$ & $23(5.98)$ & $17(73.91)$ & \\
\hline $70-79$ & $11(2.86)$ & $8(72.72)$ & \\
\hline $80-89$ & $1(0.26)$ & $0(0)$ & \\
\hline Total & 384 & $\mathbf{2 4 6}$ & \\
\hline
\end{tabular}

\section{B. Gender Distribution}

The number of female patients and male patients who attended the psychiatry OPD during study period were 207 (53.9\%) and 177 (46.1\%) respectively. The male to female ratio was 0.85 . Out of 207 female patients, only 141 (68.1\%) patients were prescribed benzodiazepines and out of 177 male patients, only 105 $(59.3 \%)$ patients were prescribed benzodiazepines [Figure 1]. There was statistically significant association between benzodiazepines use and gender distribution ( $p$-value $<0.005$ ).

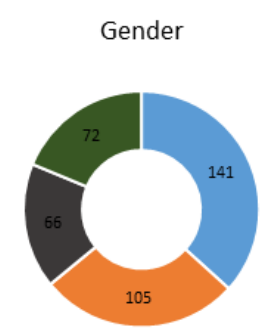

" Femalewith benzodiazepine prescription = Malewith benzodiazepine prescription

- Female without benzodiazepine prescription = Malewithout benzodiazepine prescription

Figure 1: Gender distribution with and without benzodiazepine prescription

\section{Marital status}

Out of 322 married patients, 218 patients were prescribed benzodiazepines and out of 62 unmarried patients, 28 patients were prescribed benzodiazepines. There was no statistically significant association between benzodiazepines use and marital status (' $p$ ' value $=0.346$ ) [Table 2].

Table 2: Association between marital status and the likelihood of benzodiazepines prescription.

\begin{tabular}{|l|l|l|l|}
\hline Marital Status & Number (\%) of patients & Patients prescribed with benzodiazepines (\%) & ' $p^{\prime}$ value \\
\hline Married & $322(83.85)$ & $218(67.70)$ & \multirow{2}{*}{0.346} \\
\hline Unmarried & $62(16.14)$ & $28(45.16)$ & \\
\hline
\end{tabular}

\section{Occupation Status}

Among 384 patients, all were engaged in various occupations. Most of the patients were housewives followed 
by professionals, students, skilled labour and business man. $73.38 \%$ of housewives, $49 \%$ of professionals, $45.2 \%$ of students, $63.79 \%$ of skilled labour and $28 \%$ of business man attending OPD were prescribed benzodiazepines. Association between benzodiazepines use and occupation was statistically significant (' $p$ ' value $=0.000)$ [Table 3].

Table 3: Association between occupation and the likelihood of BENZODIAZEPINESs prescription:

\begin{tabular}{|l|l|l|l|}
\hline Occupation & Number (\%) of patients & Patients prescribed with benzodiazepines (\%) & ' $\mathbf{p}^{\prime}$ value \\
\hline Business man & $41(10.7)$ & $28(68.29)$ & \\
\hline Farmer & $1(0.3)$ & $0(0)$ & \multirow{2}{*}{0.000} \\
\hline Government officer & $7(1.8)$ & $5(71.42)$ \\
\hline Housewife & $124(32.3)$ & $91(73.38)$ & \\
\hline Professional & $78(20.3)$ & $49(62.82)$ & \\
\hline Retired & $13(3.4)$ & $8(61.53)$ & \\
\hline Skilled labour & $58(15.1)$ & $37(63.79)$ & \\
\hline Student & $62(16.1)$ & $28(45.16)$ & \\
\hline Total & 384 & 246 &
\end{tabular}

\section{E. Education status}

Among 384 patients who were attended at OPD during the study time, the education level was found from no formal education upto Ph.D. $68 \%$ of patients with secondary education need benzodiazepine, followed by $63 \%$ patient with graduate level of education and $52 \%$ of patient with intermediate level of education. There was statistically significant association between benzodiazepines use and education parameter (' $p$ ' value $=0.001$ ) [Table 4]

Table 4: Association between education and the likelihood of BDZs prescription.

\begin{tabular}{|l|l|l|l|}
\hline Education & Number (\%) of patients & Patients prescribed with benzodiazepine (\%) & P - value \\
\hline No formal education & $15(3.9)$ & $10(66.67)$ & \\
\hline Primary & $53(13.8)$ & $41(77.35)$ & \multirow{2}{*}{0.001} \\
\hline Secondary & $100(26.0)$ & $68(68.0)$ & \\
\hline Intermediate & $89(23.2)$ & $52(58.42)$ & \\
\hline Graduate & $110(28.6)$ & $63(57.27)$ & \\
\hline Postgraduate & $16(4.2)$ & $12(75.0)$ & \\
\hline Ph.D. & $1(0.3)$ & $0(0)$ & \\
\hline
\end{tabular}

F. Distribution of psychiatric illness in relation with age and sex of patients prescribed with benzodiazepines

Among 384 patients attended to the OPD, only 246 patients were prescribed with benzodiazepines which were prescribed for mainly anxiety, insomnia and depression. Most prominent psychiatric illness observed in the OPD was anxiety. Between the age group 10-19 years old and 60-69 years old, both male and female were suffering from depression. Similarly, between the age group 40-49 years old, 50-59 years old and 7079 years old, both male and female were prone to anxiety. But between the age group 20-29 and 30-39 years old, most prominent psychiatric illness in male was observed depression whereas in female of 20-29 years old and 30-39 years old, it was insomnia and anxiety respectively. 


\section{G. Commonly prescribed benzodiazepines}

Out of 246 benzodiazepines prescription, clonazepam, lorazepam and chlordiazepoxide were commonly prescribed during the study period. Out of three drugs, widely prescribed drug was clonazepam (88.6\%) followed by lorazepam (4.5\%) and chlordiazepoxide (2.4\%). Least prescribed benzodiazepines were diazepam (1.6\%), alprazolam (1.6\%) and triazolam (1.2\%).

Table 5: Commonly prescribed drugs, their dose, frequency, routes of administration and duration

\begin{tabular}{|l|l|l|l|l|l|}
\hline Drug group & No. prescribed & Dose $(\mathbf{m g})$ & Frequency & Route & Duration(days) \\
\hline Clonazepam & 218 & $0.25,0.5$ & Bed time & Oral & 7 to 14 \\
\hline Lorazepam & 11 & 1 & Twice a day & Oral & 7 to 14 \\
\hline $\begin{array}{l}\text { Chlordiazepox- } \\
\text { ide }\end{array}$ & 6 & 10 & Bed time & Oral & 7 \\
\hline
\end{tabular}

$H$. Number of drugs used in patients along with benzodiazepine

Out of 246 patients prescribed with benzodiazepines, $39.1 \%$ were prescribed with one drug i.e benzodiazepine only, $56.5 \%$ and $4 \%$ patients were co-prescribed with two drugs and three or more than three drugs respectively. [Figure 1]. The other commonly co-prescribed drugs along with benzodiazepines were antidepressants, antipsychotics, antihypertensives, oral ypoglycemics and few other systemic drugs.

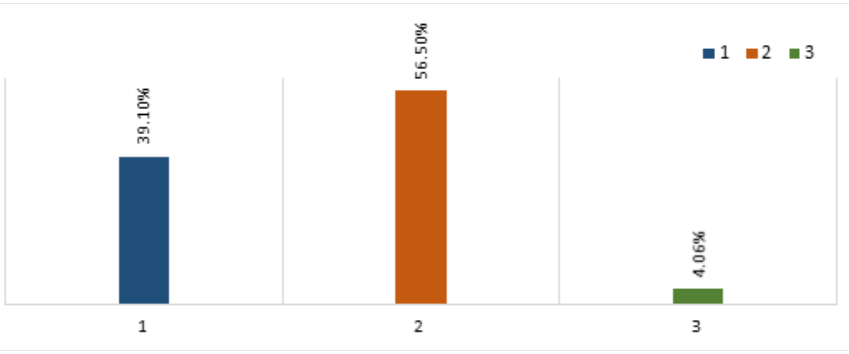

Figure 1: Number of drugs used in patients prescribed with benzodiazepines

\section{Discussion}

The present study was conducted to find out the characteristics of patient in psychiatry OPD and the utilization pattern of benzodiazepines among them. The observations from our study is able to delineate the fact that benzodiazepine use varied with patient characteristics. This study elucidated that the $64 \%$ of patients attending Psychiatric OPD were prescribed benzodiazepines which is found to be higher than the similar type of studies conducted in France (7.5\%), Lebanon (9.6\%), Sydney (19.1\%) and Chile (31.4\%). ${ }^{12-15}$

Benzodiazepines prescription were common in middle age group patients as anxiety, depression and insomnia are more common in them. ${ }^{3,4,16}$ Nepalese society is composed of various social relationships, families and relatives and depends upon the youths and adults. They express their high hopes from their adult siblings which results in anxiety and depression especially among the age group of thirty to thirty nine years. ${ }^{17}$ As the people of this age group are considered to be of productive age, in case of failure of fulfilling the hopes of the family and society, these particular age groups are prone to develop psychiatric illness. However, benzodiazepines were prescribed comparatively less to elderly patient because prescription in such patients need extra precautions. Various studies showed that they have psychomotor impairment and excessive sedation with the use of benzodiazepines. ${ }^{18-20}$

Majority of patients attending psychiatry OPD were females and $68.1 \%$ of them were prescribed benzodiazepines, slightly higher than in males (59.3\%). This may be due to the fact that females are more emotionally labile and more prone to develop insomnia and anxiety as compared to males..$^{21,22}$ The study revealed that the benzodiazepines were more commonly prescribed to housewives followed by other professionals. There was significant association between benzodiazepine use and occupation which is also supported by the results published in various 
literatures. ${ }^{23}$ Anxiety and depression is common in female housewives because of trends of female staying in home, vulnerable to stress, least contact with outer world and the male dominant culture of our society. ${ }^{24,25}$ Similarly, psychosocial factors that women experience and hormonal factors may be linked to women for higher preponderance to anxiety and depression. ${ }^{26}$ Hormone directly affect the brain chemistry that controls emotion and mood. Interestingly, there was no unemployed patients who opted to psychiatry OPD during our study period. This fact resembles the mental health unawareness among them as they might feel awkward to seek psychiatric care or they might not be aware about their abnormal mental conditions.

Marriage was found to be one of the factor that increases the likelihood of benzodiazepine use. As our study found that the higher number of married patients were attending psychiatry OPD and most of them were prescribed benzodiazepines. This finding is similar from the study conducted in United States, India and Pakistan. ${ }^{27}$ This might be due to increase in responsibility and difficulty in adjusting in family life as well as higher likelihood of misunderstanding and family disputes. All of these condition are the major risk factors for developing psychiatric illness which ultimately require the prescription of benzodiazepines. ${ }^{28,29}$

The larger number of patients attending psychiatry OPD and getting benzodiazepine as prescription were educated (96\%). Most of the patients had completed secondary to tertiary level of education. The association of education and benzodiazepine use is directly related as educated people have higher ambitions, lots of future planning and have to face different obstacles which leads to stress and frustration turning to mental illnesses like anxiety, insomnia and depression later on. These conditions required treatment and benzodiazepine is one of the commonest drug used for treatment sooner or later. $^{23}$

This study observed that the three common psychiatric illnesses in patient attending psychiatry OPD were anxiety, insomnia and depression similar to the study done by Rode SB et al. ${ }^{30}$ All these three conditions are the indications for the use of benzodiazepines. Benzodiazepine is the first line agent in anxiety and insomnia and alternative agent in depression. ${ }^{31,32}$

Among all benzodiazepines, most commonly used benzodiazepine was found to be clonazepam followed by lorazepam and chlordiazepoxide. The study conducted by Nomura $\mathrm{K}$ et al also found clonazepam as most commonly prescribed benzodiazepine. ${ }^{33}$ Various studies showed that the most commonly used benzodiazepines were bromazepam, alprazolam and temazepam. ${ }^{34-36}$ The variation in the use of benzodiazepine might be due to the differences in settings and protocols of treatment along with availability of the drug in such settings. The socio-demographic factors, ethnic factors, comorbid conditions, and the prevalence of mental illness might also play the determining role in selection of benzodiazepines. ${ }^{34,35}$ The study also showed that benzodiazepine was not always used as single agent. Antidepressant, antipsychotics, antihypertensives, oral hypoglycemics, lithium etc were co-prescribed along with benzodiazepine in over half of the patient attending psychiatry OPD. The co-prescribed drugs also sometimes play determining role in benzodiazepine selection as there might be some drug-drug interactions or some synergism which might lead to either selection of appropriate drug or appropriate dose with least interaction or more favorable clinical responses. ${ }^{37-39}$

\section{Conclusion}

The age, gender, marital status, occupational status, educational status, psychiatric illnesses and other co-morbid conditions have determining role in prescription of benzodiazepines. The prevalence of benzodiazepines use is relatively higher than that reported in the developed countries. Sensitization of the medical community and the masses of this growing problem of benzodiazepine use and associated risks is necessary to control in the developing world. This study is limited to a single OPD of tertiary care hospital so it needs further multicentric study to justify and validate the results. It is high time to standardize the use of benzodiazepines in order to fulfill the rationality of prescription with safeguarding the mental health in wider sense. 


\section{References}

1. Grover S, Avasthi A, Sinha V, Lakdawala B, Bathla $M$, Sethi $S$, et al. Indian Psychiatric Society multicentric study: Prescription patterns of psychotropics in India. Indian J Psychiatry. 2014;56:253-64.

2. Weich S, Pearce HL, Croft P, et al. Effect of anxiolytic and hypnotic drug prescriptions on mortality hazards: retrospective cohort study. The BMJ. 2014;348:g1996. doi:10.1136/ bmj.g1996.

3. Hollister LE, Müller-Oerlinghausen B, Rickels K, Shader RI. Clinical uses of benzodiazepines. J Clin Psychopharmacol. 1993 Dec;13(6 Suppl 1):1S-169S.

4. Lapierre YD. Introduction. The multiplicity of uses of benzodiazepines. Can J Psychiatry. 1993 Nov;38 Suppl 4:S101.

5. Gallagher HC. Addressing the Issue of Chronic, Inappropriate Benzodiazepine Use: How Can Pharmacists Play a Role? Pharmacy 2013, 1, 65-93; doi:10.3390/pharmacy1020065

6. Lader M, Tylee A, Donoghue J. Withdrawing benzodiazepines in primary care. CNS Drugs 2009, 23, 19-34.

7. Donoghue, J, Lader $M$. Usage of benzodiazepines: A review. Int. J. Psychiatr. Clin. Prac. 2010, 14, 78-87.

8. Arbanas, G.; Arbanas, D.; Dujam, K. Adverse effects of benzodiazepines in psychiatric outpatients. Psychiatr. Danub. 2009, 21, 103107.

9. FDA. Paving the Way for Personalized Medicine: FDA's Role in a New Era of Medical Product Development. Oct 2013. Available from: https://www.fda.gov/ downloads/scienceresearch/specialtopics/ personalizedmedicine /ucm372421.pdf

10. Kuperman GJ, Bobb A, Payne $T H$, et al. Medication-related Clinical Decision Support in Computerized Provider Order Entry Systems: A Review. Journal of the American Medical Informatics Association: JAMIA. 2007;14(1):29-40. doi:10.1197/jamia.M2170.

11. Heather N, Bowie A, McAvoy B, et al. Randomised controlled trial of two brief interventions against long-term benzodiazepine use: outcome of intervention. Addiction Research and Theory. April 2004, Vol. 12, No. 2, pp. 141-154

12. Ramadan WH, El Khoury GM, Deeb ME, Sheikh-Taha M. Prescription patterns of benzodiazepines in the Lebanese adult population: a cross-sectional study. Neuropsychiatric Disease and Treatment. 2016;12:2299-2305. doi:10.2147/ NDT.S113078

13. Ruiz I, Offermanns J, Lanctôt KL, Busto U. Comparative study on benzodiazepine use in Canada and Chile. J Clin Pharmacol. 1993 Feb;33(2):124-9.

14. Lagnaoui R, Moore N, Longy-Boursier $M$, Baumevieille $M$, Bégaud $B$. Benzodiazepine use in patients hospitalized in a department of internal medicine: frequency and clinical correlates. Pharmacoepidemiol Drug Saf. 2001 Oct-Nov;10(6):531-5.

15. Howes JB, Ryan J, Fairbrother G, O'Neill $\mathrm{K}$, Howes LG. Benzodiazepine prescribing in a Sydney teaching hospital. Med J Aust. 1996 Sep 16;165(6):305-8.

16. Olfson $M$, King $M$, Schoenbaum $M$. JAMA Psychiatry. Benzodiazepine use in United States. 2015 Feb;72(2):136-42. doi: 10.1001/ jamapsychiatry.2014.1763.

17. Goldstein MC, Schuler S, Ross JL. Social and economic forces affecting intergenerational relations in extended families in a third world country: A cautionary tale from South Asia. Journal of Gerontology. 1983;38 (6):716-724.

18. Benzodiazepines: Revisiting Clinical Issues in Treating Anxiety Disorders. Primary Care Companion to The Journal of Clinical Psychiatry. 2005;7(1):23-32.

19. Roy-Byrne PP. The GABA-benzodiazepine receptor complex in anxiety. pp. 1568-1569.In: Rosenbaum JF, chair. Utilizing Benzodiazepines in Clinical Practice: An Evidence-Based Discussion [Acadmemic Highlights]. J Clin Psychiatry. 2004 65: 1565-1574.

20. Wang PS, Bohn RL, and Glynn RJ. et al. Hazardous benzodiazepine regimens in the elderly: effects of half-life dosage, and duration on the risk of hip fracture. Am J Psychiatry. 2001 158:892-898. 
21. Bitsika V, Sharpley CF, Melhem TC. Gender Differences in Factor Scores of Anxiety and Depression among Australian University Students: Implications for Counselling Interventions. Canadian Journal of Counselling .2010;44(1): 51-64

22. Bohra N, Srivastava S, Bhatia MS. Depression in women in Indian context. Indian Journal of Psychiatry. 2015;57(Suppl 2):S239-S245. doi:10.4103/0019-5545.161485.

23. Ahmer S, Salamat S, Khan AMR, Iqbal PS, Haider II et al. Patterns of Benzodiazepines use in Psychiatric outpatients in Pakistan: a cross sectional study. Bio Med central.2009; 5(9):16.

24. Straussner SL, Zelvin E. Gender and addictions: Men and women in treatment. Jason Aronson; 1997.

25. Leach LS, Christensen $H$, Mackinnon AJ, Windsor TD, Butterworth P. Gender differences in depression and anxiety across the adult lifespan: the role of psychosocial mediators. Social psychiatry and psychiatric epidemiology. 2008 Dec 1;43(12):983-98.

26. Piccinelli M, Wilkinson G. Gender differences in depression. The British Journal of Psychiatry Dec 2000, 177 (6) 486-492; DOI: 10.1192/ bjp.177.6.486

27. Patel MJ, Ahmer S, Khan F, et al. Benzodiazepine use in medical out-patient clinics: a study from a developing country. J Pak Med Assoc. 2013; 63 (6):717-720.

28. Susser E, Schwartz S, Morabia A, Bromet EJ. Psychiatric epidemiology: searching for the causes of mental disorders. Oxford University Press; 2006 Jun 1.

29. Crowe M. Couples and mental illness. Sexual and relationship therapy. 2004 Aug 1;19(3):309-18.

30. Rode SB, Ajagallary RK, Salankar HV and Sinha U. A study on drug prescription pattern in psychiatry outpatient department from a tertiary care teaching hospital. International Journal of Basic and clinical Pharmacology.2014; 3(3): 517-522.

31. Cascade E and Kalali AH. Use of benzodiazepine in the treatment of anxiety. US National Library of Medicine.2008; 5(9):21-29.

32. Sweileh W and Jaradat N. Pharmacotherapeutic implications and prescribing pattern of Benzodiazepines by psychiatrists and Neurologists. An- Najah Univ.J.Res.2004; 18(1):25-38.

33. Nomura K, Nakao M, Sato M, Yano E. Regular prescriptions for benzodiazepines: a cross sectional study of outpatients at a university hospital.PMID.2006; 45(22):1279-1283.

34. Alvarenga JM, Loyola FL, Firmo JO, LimaCosta MF, Uchoa E. Prevalence and sociodemographic characteristics associated with benzodiazepines use among community dwelling older adults: the Bambui Health and Aging Study. Rev Bras Psiquiatr. 2008; 30(1):711.

35. Gleason PP, Schulz R, Nicholas L, Newsom JT et al. Correlates and prevalence of benzodiazepines use in community- dwelling elderly. J Gen Intern Med.1998; 13:243-250.

36. Shan K, Nolan JA, Turner P and Jackson SHD. Prescription of benzodiazepines in a London teaching hospital. Journal of the Royal Society of Medicine.1990; 83:307-308.

37. Tiihonen J, Suokas JT, Suvisaari JM, Haukka J,KorhonenP.Polypharmacy withantipsychotics, antidepressants, or benzodiazepines and mortality in schizophrenia. Arch Gen Psychiatry. 2012 May;69(5):476-83. doi: 10.1001/archgenpsychiatry.2011.1532.

38. Abernethy DR, Greenblatt DJ, Ochs HR, Shader RI. Benzodiazepine drug-drug interactions commonly occurring in clinical practice. Curr Med Res Opin. 1984;8 Suppl 4:80-93.

39. Tanaka E. Clinically significant pharmacokinetic drug interactions with benzodiazepines. Journal of clinical pharmacy and therapeutics. 1999 Oct. 24: 347-355. doi:10.1046/j.13652710.1999.00247.x 